\title{
Role of heme oxygenase-1 in demethylating effects on SKM-1 cells induced by decitabine
}

\author{
R. Gao ${ }^{1,2,3}$, D. Ma ${ }^{1,2,3,4}$, P. Wang ${ }^{1,2,3}$, J. Sun ${ }^{6}$, J.S. Wang ${ }^{1,2,3}$ and Q. Fang ${ }^{4,5}$ \\ ${ }^{1}$ Department of Hematology, Affiliated Hospital of Guiyang Medical University, \\ Guiyang, China \\ ${ }^{2}$ Key Laboratory of Hematological Disease Diagnostic \& Treat Centre of Guizhou Province, \\ Guiyang, China \\ ${ }^{3}$ Guizhou Province Hematopoietic Stem Cell Transplantation Center, \\ Affiliated Hospital of Guiyang Medical University, Guiyang, China \\ ${ }^{4}$ Department of Pharmacy, Affiliated Baiyun Hospital of Guiyang Medical University, \\ Guiyang, China \\ ${ }^{5}$ Department of Pharmacy, Affiliated Hospital of Guiyang Medical University, \\ Guiyang, China \\ ${ }^{6}$ School of Pharmacy, Guiyang Medical University, Guiyang, China \\ Corresponding author: J.S. Wang \\ E-mail: wangjishi9646@163.com \\ Genet. Mol. Res. 14 (4): 17788-17798 (2015) \\ Received September 10, 2015 \\ Accepted November 14, 2015 \\ Published December 22, 2015 \\ DOI http://dx.doi.org/10.4238/2015.December.22.3
}

ABSTRACT. We evaluated the influence of heme oxygenase-1 (HO1) gene inhibition in myelodysplastic syndrome (MDS) cell line SKM-1 on enhancement of the demethylating effects of decitabine on p15, and explored the possible mechanism. DNMT1 gene expression in SKM-1 cells was silenced by being transfected by a constructed siRNA with liposomes. The proliferation inhibition rates after drug treatment were detected by cell counting kit-8 assay. The apoptotic rates were detected by Annexin V/PI assay with flow cytometry. The expressions of p16, p15, TP73, CDH1, ESR1, and PDLIM4 mRNAs were detected by real-time PCR, and those of HO-1, DNMT1, DNMT3A, DNMT3B, HDAC, and p15 proteins were measured by western blot. The degree of methylation of the $p 15$ gene was analyzed by 
using methylation-specific PCR (MSP). CCK-8 assay showed that after $\mathrm{HO}-1$ gene expression was inhibited; the proliferation rate of SKM-1 cells treated by decitabine $(70.91 \pm 0.05 \%)$ was significantly higher than that of the control group (53.67 $\pm 0.05 \%$ ). Flow cytometry showed that the apoptotic rate of SKM1 cells treated by decitabine in combination with $\mathrm{HO}-1$ expression inhibition $(44.25 \pm 0.05 \%)$ exceeded that of the cells treated by this drug alone (37.70 \pm $0.05 \%)$. MSP showed that inhibiting HO-1 expression significantly increased the degree of methylation of the $p 15$ gene. As suggested by western blot, the degree of methylation of the $\mathrm{p} 15$ protein was changed after decitabine treatment when the expression of the HO-1 protein was changed, being associated with the affected DNMT1 expression. Inhibited HO-1 expression attenuated the hypermethylation of CDKN2B by suppressing DNMT1, which was conducive to treatment by cooperating with decitabine. In conclusion, the findings of this study provide valuable experimental evidence for targeted MDS therapy, and a theoretical basis for further studies.

Key words: Myelodysplastic syndrome; Heme oxygenase-1; Methyltransferase; p15

\section{INTRODUCTION}

As a heterogeneous group of hematopoietic stem cell-related clonal diseases, myelodysplastic syndrome (MDS) is typified by myeloid dysplasia, ineffective hematopoiesis, increasing number of primitive cells, and high risk of transformation to acute myelocytic leukemia (AML). MDS is clinically manifested as an increase in hematopoietic cell number, accompanied by monolineage or multilineage dysplasia involving one or more abnormal myeloid cell types, and peripheral blood cytopenias. The pathogenesis of MDS remains unclear, and has been attributed to exogenous and endogenous factors (Dalamaga et al., 2002; Nimer, 2008; Vardiman et al., 2009; Bejar et al., 2011). Most patients have karyotype abnormalities (Ebert et al., 2008); some of these are affected with mutations in the oncogenes and anti-oncogenes, as well as aberrant expressions of apoptosis-related genes (Kao et al., 2011; Fuchs, 2012). Epigenetic changes involving DNA methylation are also correlated with the onset of hematological tumors (Olk-Batz et al., 2011).

At the molecular level, MDS is a result of malignant cell clones arising because of silenced expressions of anti-oncogenes after the demethylation of anti-oncogene promoters and mutations in the relevant regulatory genes (Wang et al., 2011). For instance, anti-oncogenes such as p15 are deactivated because of over-methylation, leading to cell cycle abnormalities, which, together with increased cell proliferation and reduced apoptosis and differentiation, finally induce the generation of tumor clones (Fandy et al., 2009). p15, which belongs to the cyclin-dependent kinase inhibitor (CDKI) family, codes for the p15 protein that directly binds to CDK4, thereby blocking the binding of CDK to cyclin D, and subsequently arresting the cells at the G1/S transition phase (Rual et al., 2005). Kim et al. (2013) reported that CDKN2B was ubiquitously hypermethylated in children and adults suffering from MDS. Zhang et al. (2013) also discovered that the MDS patients treated with low doses of decitabine in addition to daunorubicin and cytarabine showed a significantly better prognosis, compared to those treated individually 
with decitabine. In addition, demethylation was observed to play an important role in the clinical treatment of MDS. However, the detailed mechanism remains to be clarified. Since this disease is particularly sensitive to DNA methyltransferase inhibitors, DNA methylation is believed to be vital for its onset (Zhang et al., 2013).

Heme oxygenase-1 (HO-1), an isozyme of heme oxygenase, is the rate-limiting enzyme of the heme catabolic process, and can be expressed upon being induced by multiple external factors. Under disease or stress conditions, HO-1 protects the cells from apoptosis, promotes cell proliferation, and mitigates the inflammatory response (Ryter et al., 2006; Alam and Cook, 2007). The HO-1 gene has been associated with the progression of AML (Miyazaki et al., 2010; Ma et al., 2014). In addition, it has been known to shield the AML cells from tumor necrosis factorinduced apoptosis through the regulation of the transcription factors Nrf2, NF-kB, and AP-1, and the reduction in reactive oxygen species accumulation in these cells (Fang et al., 2003; Heasman et al., 2011). It has also been reported that the Fas-associated death domain-like IL-1-converting enzyme-like inhibitory protein contributed to the resistance of AML cells to apoptosis, via the regulation of HO-1 expression (Rushworth et al., 2010).

We have previously discovered high $\mathrm{HO}-1$ expression in MDS patients with CDKN2B methylation (and vice versa). The treatment of SKM-1 cells with decitabine led to the downregulation of $\mathrm{HO}-1$ expression, in addition to CDKN2B demethylation. Therefore, we postulated a correlation between the $\mathrm{HO}-1$ gene and MDS progression and over-methylation of tumor suppressor gene promoters, aiming to prove that the $\mathrm{HO}-1$ gene can function as a potential target for MDS therapy.

\section{MATERIAL AND METHODS}

\section{Cell line and culture conditions}

The SKM-1 cell line was purchased from the Japanese Collection of Research Bioresources, and cultured in Dulbecco's modified Eagle's medium and F12, supplemented with 15\% fetal bovine serum, $100 \mathrm{U} / \mathrm{mL}$ penicillin, and $100 \mathrm{mg} / \mathrm{mL}$ streptomycin. All reagents and antibiotics were obtained from Thermo Scientific Fisher (Waltham, MA, USA). All cells were maintained in a $37^{\circ} \mathrm{C}$ incubator with $95 \%$ humidity and $5 \% \mathrm{CO}_{2}$.

\section{Construction of DNMT1 siRNA}

SiRNA was constructed to transfect the cells for the knockdown of DNMT1 expression. The siRNA sequences specific for DNMT1 were GGAUGAGAAGAGACGUAGAtt, UCUACGUCUCUUCUCAUCCtg, GCACCUCAUUUGCCGAAUAtt, and UAUUCGGCAAAUGAGG UGCtg.

\section{Cell proliferation inhibition assay}

The cells were inoculated onto 6 -well plates at the density of $10^{8} \mathrm{cells} / \mathrm{mL}$, and incubated with different doses of ZnPP IX and Hemin, in combination with decitabine, for 24 and $48 \mathrm{~h}$. The inhibitory effects were determined using the cell counting kit-8 (CCK-8) (Beyotime Institute of Biotechnology, Shanghai, China) as per the manufacturer protocols. 


\section{Western blot}

The cells were collected, lysed with western and IP lysates as well as $10 \mathrm{X}$ phenylmethanesulfonyl fluoride solution, incubated on ice for $20 \mathrm{~min}$, and centrifuged at $4^{\circ} \mathrm{C}$ and $12,000 \mathrm{rpm}$ for $10 \mathrm{~min}$. The protein concentrations were measured using a microplate reader, and the sodium dodecyl sulfate (SDS) loading buffer was added $10 \mathrm{~min}$ prior to the boiling water treatment. The protein bands obtained after separation by SDS polyacrylamide gel electrophoresis $(10 \%$ SDS-PAGE) were transferred to a nitrocellulose membrane, incubated with the HO-1, p15, DNMT1, DNMT3A, DNMT3B, HDAC antibodies, and detected with electrochemiluminescent substrates (for western blotting). All reagents were purchased from Beyotime Institute of Biotechnology.

\section{Real-time fluorescent quantitative polymerase chain reaction (PCR)}

The cells were collected and treated with (shaken) a mixture of TRIzol reagent (Beyotime Institute of Biotechnology) and chloroform. This mixture was then allowed to stand still, and subsequently centrifuged at $4^{\circ} \mathrm{C}$ and $12,000 \mathrm{rpm}$ for $15 \mathrm{~min}$. The supernatant was mixed with an equal volume of isopropanol, allowed to stand still for $20 \mathrm{~min}$, and centrifuged again at $4^{\circ} \mathrm{C}$ and $12,000 \mathrm{rpm}$ for $10 \mathrm{~min}$. Afterwards, the resulting total RNA was washed with $75 \%$ ethanol and reverse-transcribed to cDNA with Revert Aid First-Strand cDNA Synthesis Kit (Thermo Scientific Fisher). The obtained cDNA was used for real-time quantitative PCR, using primers provided by Airui Technology Corporation (Guiyang, China) and iQ SYBR Green supermix (Bio-Rad, Hercules, CA, USA).

\section{Methylation-specific PCR (MSP)}

The cells were collected; the total DNA extracted using the TIANamp Blood DNA Kit (Tiangen Biotech Co., Ltd., Beijing, China), and the samples modified with the BisulFlash ${ }^{\mathrm{TM}}$ DNA Modification Kit (Epigentek Group Inc.). Primers specific for methylation and demethylation were designed and amplified, and the products were subjected to agarose gel electrophoresis. The designed primers were as follows: methylation, forward 5'-TTACGAGGGGCGGTCGTACGCGGG-3' and reverse 5'-AAAACGACCGACGCGAACGCCTCC-3'; and demethylation, forward 5'-TTATGAGGGGTGGT TGTATGTGGG-3' and reverse 5'-AAAACAACCAACACAAACACCTCC-3'.

\section{Detection of cell apoptosis using the Annexin V/PI staining assay}

The cells from the control-, Hemin-, and ZnPP IX-treated groups were collected, washed twice with PBS, and counted. Subsequently, $10^{7}$ cells of each group were suspended with 500 $\mu \mathrm{L} 1 \mathrm{X}$ binding buffer, into which was added $5 \mu \mathrm{L}$ Annexin V-fluorescein isothiocyanate and $5 \mu \mathrm{L}$ $\mathrm{PI}$. These treated cells were placed in the dark for $15 \mathrm{~min}$, and subsequently analyzed by flow cytometry after $30 \mathrm{~min}$.

\section{RESULTS}

\section{Upregulation of HO-1 protects the SKM-1 cell line against decitabine-induced apoptosis via the inhibition of $\mathrm{p} 15$}

The endogenous expression of the HO-1 gene in SKM-1 cells was successfully up- 
regulated by the Hemin enhancer, and downregulated by the ZnPP IX inhibitor. The control-, Hemin-, and ZnPP IX-treated groups were co-cultured with different doses of decitabine. The increase in decitabine dosage led to sensitivity of SKM-1 cell inhibition. The inhibitory effects of decitabine were less obvious on the HO-1 upregulation group, compared to those on the control group. However, these effects were more evident in the downregulation group. Meanwhile, of the detected antioncogenes, only p15 was differentially expressed in the control and two of the experimental groups (treated with decitabine). The increase in the $\mathrm{HO}-1$ protein led to a corresponding decrease in $\mathrm{p} 15$ expression, and vice versa (Figure 1).

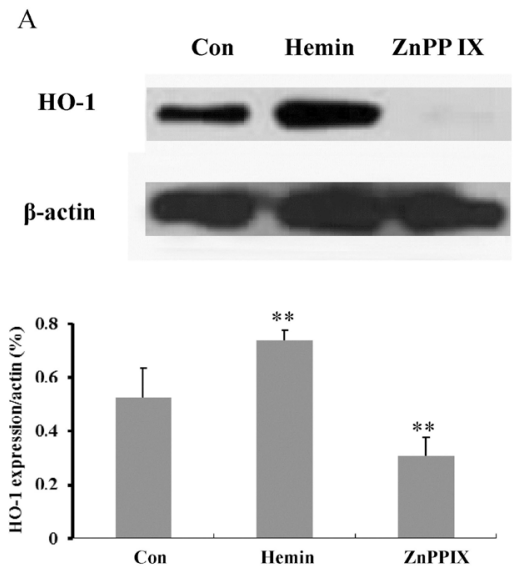

C

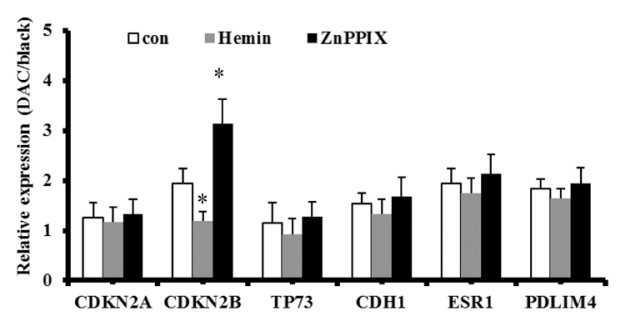

B

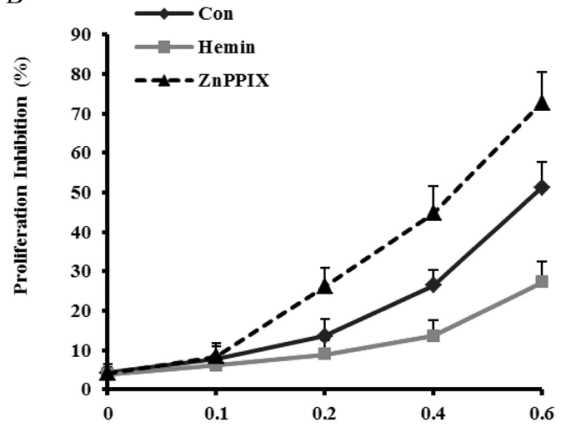

Concentration of DAC $(\mu \mathrm{M})$

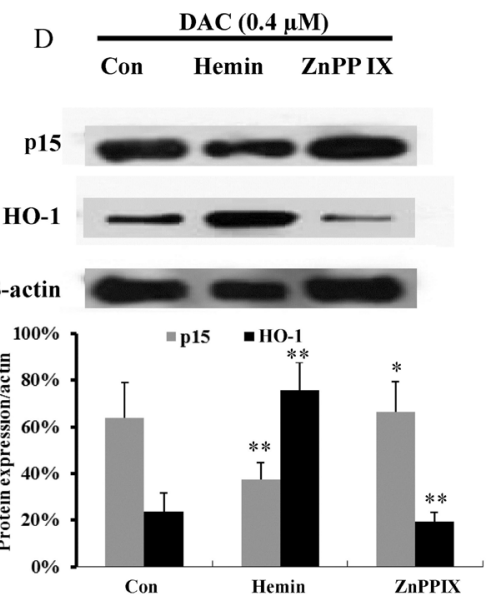

Figure 1. Regulation of HO-1 expression affecting the inhibitory effects of decitabine on SKM-1 cells and p15 expression. A. Endogenous HO-1 expression upregulated and downregulated by Hemin and ZnPP IX, respectively. B. Experimental groups co-cultured with $0,0.1,0.2,0.4$, and $0.6 \mu \mathrm{M}$ decitabine. The SKM-1 cells were inhibited with greater efficiency upon increasing the dosage of decitabine. Decitabine-induced inhibition was less obvious in $\mathrm{HO}-1$ upregulated cells compared to the cells of the control group. On the other hand, decitabine exerted a greater inhibitory effect on the HO-1 downregulated cells compared to the cells of the control group. C. Anti-oncogenes CDKN2A, CDKN2B, TP73, CDH1, ESR1, and PDLIM4 detected by real-time PCR; however, only CDKN2B, coding for the antioncogene $\mathrm{p} 15$, was differentially expressed. D. Cells treated with $0.4 \mu \mathrm{M}$ decitabine. The results of the western blot analysis revealed that $\mathrm{p} 15$ expression was upregulated and downregulated when $\mathrm{HO}-1$ expression was inhibited or promoted, respectively. Con $=$ control. 


\section{HO-1 upregulation resulted in a reduced level of decitabine-induced p15 demethylation, by increasing the DNMT1 expression}

The cell groups were treated with placebo and decitabine. The levels of methylation of p15 gene were elevated upon upregulation of the HO-1 gene, as detected by MSP. The HO-1 upregulation group resisted the demethylating effects of decitabine, while the control and HO-1 downregulation group did not. In addition, DNMT1 was differentially expressed in all groups. The expression of DNMT1 was observed to increase and decrease with the upregulation and downregulation of HO-1, respectively. In contrast, the cell cycle initiation factor- and anti-oncogene methylation-related gene expression (i.e., DNMT3A, DNMT3B and HDAC) was similar in all groups (Figure 2).
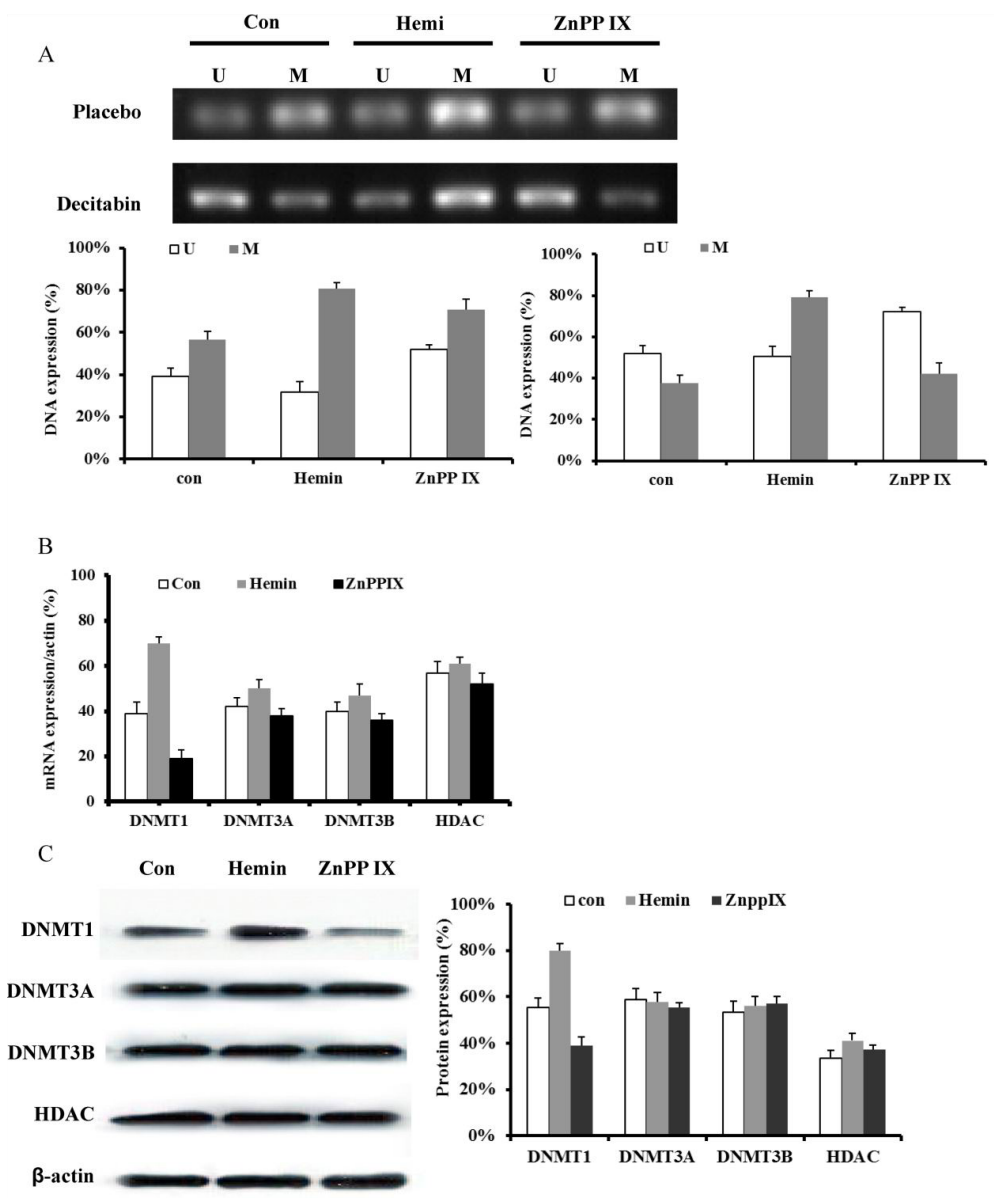

Figure 2. HO-1 affecting p15 methylation by altering the DNMT1 expression. A. MSP revealed an increase in p15 demethylation upon upregulation of $\mathrm{HO}-1$ expression. The treatment of cells with decitabine resulted in a reduction in the demethylation in the HO-1 upregulated group; however, the control and $\mathrm{HO}-1$ downregulated groups did not show any such decrease. B. Real-time PCR of DNA methylation-related genes showing significant differences in DNMT1 expression following HO-1 regulation. C. Western blot analysis revealing a decrease (or increase) in DNMT1 expression corresponding to the increase (or decrease) in $\mathrm{HO}-1$ expression. Con $=$ control. 


\section{Inhibition of DNMT1 attenuated the effects of HO-1 on p15 expression}

Following inhibition (using siRNA in combination with $\mathrm{HO}-1$ regulation), DNMT1 protein expression was observed to be lower than in the group wherein only the HO-1 protein was regulated. This resulted in an increase in p15 protein expression, suggesting that changes in $\mathrm{HO}-1$ expression affected p15 expression by altering DNMT1 expression. Similarly, MSP revealed that the demethylating effect of decitabine on the group with inhibited DNMT1 expression was greater than that on the group without inhibited DNMT1 expression (Figure 3).

A

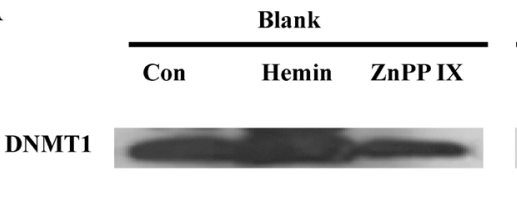

HO-1

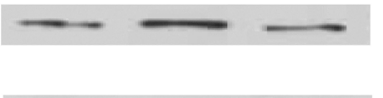

P15
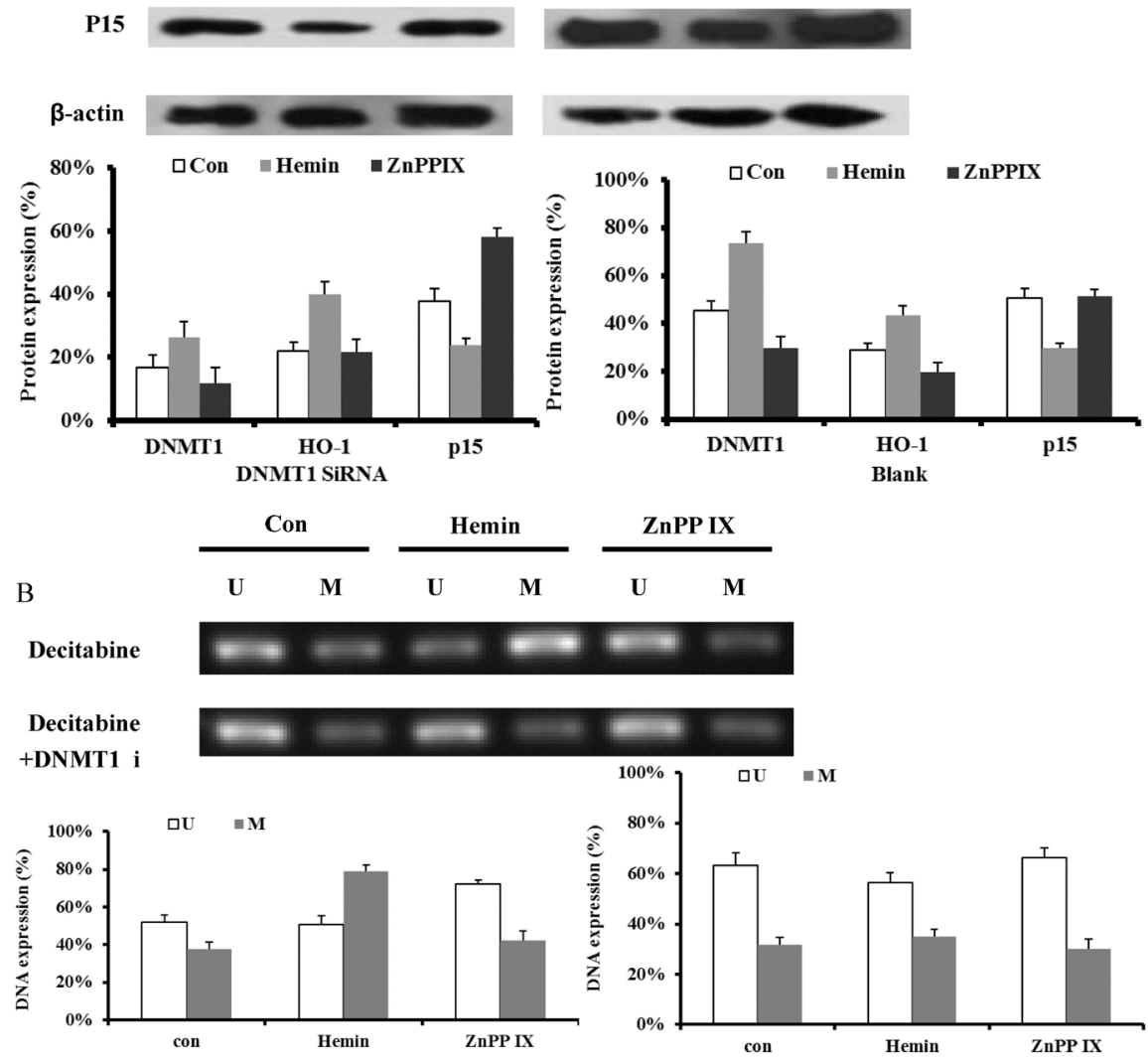

Figure 3. Inhibition of DNMT1 conducive to $\mathrm{p} 15$ expression. A. Western blot analysis performed following the inhibition of DNMT1 expression by treatment with small interfering RNA (siRNA) revealing a moderate change in the HO-1 protein expression and an increase in the 15 protein expression. B. MSP showing that the inhibition of DNMT1 expression boosted the demethylating effects of decitabine. Con $=$ control. 


\section{Inhibition of HO-1 induced SKM-1 cell apoptosis via the reduction of DNMT1 expression}

The regulation of $\mathrm{HO}-1$ expression led to an increase in the apoptotic rate of the cells treated with decitabine compared to the untreated group. In addition, the combination of DNMT1 inhibition and decitabine treatment augmented the apoptotic rate, compared to that caused by decitabine alone. Therefore, DNMT1 inhibition weakened the over-methylation of tumor cells and enhanced the demethylating effects of decitabine, which ultimately facilitated cell apoptosis (Figure 4).
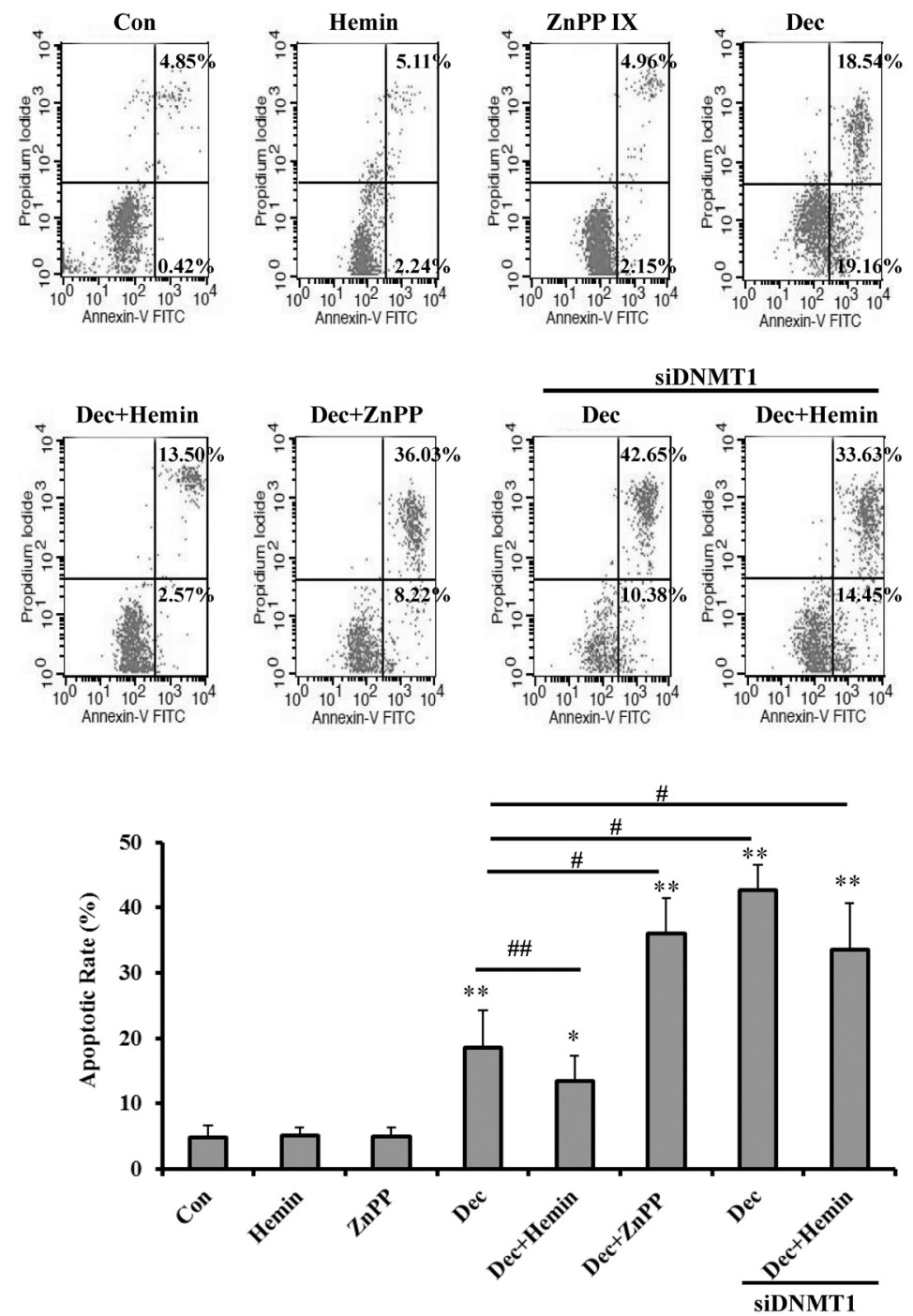

Figure 4. Reduction in HO-1 over-expression facilitated tumor cell apoptosis. Apoptosis rate of un-transfected/ transfected cells treated with Hemin/ZnPP IX and decitabine detected using flow cytometry. All experiments were repeated thrice. ${ }^{* *} \mathrm{P}<0.01$; ${ }^{*} \mathrm{P}<0.05 ;{ }^{\# P}<0.01$; and ${ }^{\#} \mathrm{P}<0.05$. Con $=$ control; Dec $=$ decitabine. 


\section{DISCUSSION}

So far, the onset and progression of MDS has been mainly ascribed to over-methylation (Griffiths and Gore, 2013). In this study, we have attempted to clarify the in vitro relationship between $\mathrm{HO}-1$ and methylation, and analyze the possible mechanism by regulating the endogenous expression of the HO-1 gene using an inhibitor, in order to verify the applicability of $\mathrm{HO}-1$ as a potential target for MDS therapy, as well as to discover novel treatment protocols.

The studies conducted so far have correlated $\mathrm{HO}-1$ expression with AML or CML, but never with MDS. Specifically, the mechanism governing the relationship between $\mathrm{HO}-1$ and methylation has not been unraveled so far. MDS is currently being treated by chemotherapy and hematopoietic stem cell transplantation; however, these methods have serious drawbacks, highlighting the need for targeted therapy. The aim of this study was to confirm the participation of HO-1 in the regulation of anti-oncogene promoter methylation. We also attempted to provide experimental proof that the downregulation of HO-1 sensitized MDS toward demethylation, and a theoretical basis for the use of $\mathrm{HO}-1$ as a potential target for the treatment of MDS.

The inhibition of HO-1 expression significantly affected the therapeutic effects of decitabine on MDS. Decitabine (2'-deoxy-5-azacytidine) is known to exert a demethylating effect via the suppression of both DNA methyltransferase activity and over-methylation of antioncogenes, thereby promoting cell differentiation and apoptosis (Li et al., 2014; Martinez-Galan et al., 2014). Decitabine reactivates the anti-oncogenes for MDS, by reducing their methylation levels. However, the relationship between decitabine and the recovery of p15 gene expression (Daskalakis et al., 2002; Raj et al., 2007) remains a controversial topic. In general, decitabine cannot effectively treat human MDS under conditions of high $\mathrm{HO}-1$ expression. In this study, the DNA methyltransferase expression was augmented in addition to high HO-1 gene expression, which resisted the demethylating effects of decitabine. This resulted in over-methylation and deactivation of the anti-oncogene $\mathrm{p} 15$. Subsequently, the tumor cells became prone to proliferation. On the contrary, inhibition of the endogenous HO-1 expression by ZnPP IX led to a reduction in DNA methyltransferase expression, subsequently resulting in an increase in $p 15$ gene methylation. The application of decitabine under these conditions resulted in easier tumor cell differentiation and apoptosis. The simultaneous inhibition of HO-1 and DNMT1 expressions was observed to further enhance the demethylating effects of decitabine, and raise the apoptotic rates of tumor cells.

In addition to shielding the cells from a variety of stimuli and inflammations, HO-1 is known to protect normal tissues as well as cancer cells (Choi et al., 2014; Tertil et al., 2014). In summary, we theorize that MDS patients with barely alleviated symptoms may have high levels of $\mathrm{HO}-1$ expression. Further studies are still ongoing in our group to validate this presumption, as well as to clarify the pathway with which $\mathrm{HO}-1$ affects DNA methyltransferase, and to provide an experimental basis and possible therapeutic target for epigenetic modification of MDS.

\section{Conflicts of interest}

The authors declare no conflict of interest.

\section{ACKNOWLEDGMENTS}

Research supported in part by the National Natural Science Foundation of China (\#81070444, \#81270636, \#81360501, and \#81470006), the International Cooperation Project of 
GuiZhou Province (\#2011-7010), the Social Project of GuiZhou Province (\#2011-3012), and the Provincial Governor Special Fund of GuiZhou Province (\#2010-84).

\section{REFERENCES}

Alam J and Cook JL (2007). How many transcription factors does it take to turn on the heme oxygenase-1 gene? Am. J. Respir. Cell Mol. Biol. 36: 166-174.

Bejar R, Levine R and Ebert BL (2011). Unraveling the molecular pathophysiology of myelodysplastic syndromes. J. Clin. Oncol. 29: 504-515.

Choi JH, Jin SW, Han EH, Park BH, et al. (2014). Platycodon grandiflorum root-derived saponins attenuate atopic dermatitislike skin lesions via suppression of NF-KB and STAT1 and activation of Nrf2/ARE-mediated heme oxygenase-1. Phytomedicine 21: 1053-1061.

Dalamaga M, Petridou E, Cook FE and Trichopoulos D (2002). Risk factors for myelodysplastic syndromes: a case-control study in Greece. Cancer Causes Control 13: 603-608.

Daskalakis M, Nguyen TT, Nguyen C, Guldberg P, et al. (2002). Demethylation of a hypermethylated P15/INK4B gene in patients with myelodysplastic syndrome by 5-Aza-2'-deoxycytidine (decitabine) treatment. Blood 100: 2957-2964.

Ebert BL, Pretz J, Bosco J, Chang CY, et al (2008). Identification of RPS14 as a 5q- syndrome gene by RNA interference screen. Nature 451: 335-339.

Fandy TE, Herman JG, Kerns P, Jiemjit A, et al. (2009). Early epigenetic changes and DNA damage do not predict clinical response in an overlapping schedule of 5-azacytidine and entinostat in patients with myeloid malignancies. Blood 114: 2764-2773.

Fang J, Sawa T, Akaike T, Akuta T, et al. (2003). In vivo antitumor activity of pegylated zinc protoporphyrin: targeted inhibition of heme oxygenase in solid tumor. Cancer Res. 63: 3567-3574.

Fuchs $\mathrm{O}$ (2012). Important genes in the pathogenesis of 5q- syndrome and their connection with ribosomal stress and the innate immune system pathway. Leuk. Res. Treat. 179402.

Griffiths EA and Gore SD (2013). Epigenetic therapies in MDS and AML. Adv. Exp. Med. Biol. 754: 253-283.

Heasman SA, Zaitseva L, Bowles KM, Rushworth SA, et al. (2011). Protection of acute myeloid leukaemia cells from apoptosis induced by front-line chemotherapeutics is mediated by haemoxygenase-1. Oncotarget 2: 658-668.

Kao HW, Sanada M, Liang DC, Lai CL, et al. (2011). A high occurrence of acquisition and/or expansion of C-CBL mutant clones in the progression of high-risk myelodysplastic syndrome to acute myeloid leukemia. Neoplasia 13: 1035-1042.

Kim M, Kook H, Park HJ, Ahn HS, et al. (2013). Quantitative comparison of CDKN2B methylation in pediatric and adult myelodysplastic syndromes. Acta Haematol. 130: 115-121.

Li YX, Lu Y, Li CY, Yuan P, et al. (2014). Role of CDH1 promoter methylation in colorectal carcinogenesis: a meta-analysis. DNA Cell Biol. 33: 455-462.

Ma D, Fang Q, Li Y, Wang J, et al. (2014). Crucial role of heme oxygenase-1 in the sensitivity of acute myeloid leukemia cell line Kasumi-1 to ursolic acid. Anticancer Drugs 25: 406-414.

Martínez-Galán J, Torres-Torres B, Núñez MI, Lopez-Penalver J, et al. (2014). ESR1 gene promoter region methylation in free circulating DNA and its correlation with estrogen receptor protein expression in tumor tissue in breast cancer patients. BMC Cancer 14: 59.

Miyazaki T, Kirino Y, Takeno M, Samukawa S, et al. (2010). Expression of heme oxygenase-1 in human leukemic cells and its regulation by transcriptional repressor Bach1. Cancer Sci. 101: 1409-1416.

Nimer SD (2008). Myelodysplastic syndromes. Blood 111: 4841-4851.

Olk-Batz C, Poetsch AR, Nöllke P, Claus R, et al. (2011). Aberrant DNA methylation characterizes juvenile myelomonocytic leukemia with poor outcome. Blood 117: 4871-4880.

Raj K, John A, Ho A, Chronis C, et al. (2007). CDKN2B methylation status and isolated chromosome 7 abnormalities predict responses to treatment with 5-azacytidine. Leukemia 21: 1937-1944.

Rual JF, Venkatesan K, Hao T, Hirozane-Kishikawa T, et al. (2005). Towards a proteome-scale map of the human proteinprotein interaction network. Nature 437: 1173-1178.

Rushworth SA, Zaitseva L, Langa S, Bowles KM, et al. (2010). FLIP regulation of HO-1 and TNF signalling in human acute myeloid leukemia provides a unique secondary anti-apoptotic mechanism. Oncotarget 1: 359-366.

Ryter SW, Alam J and Choi AM (2006). Heme oxygenase-1/carbon monoxide: from basic science to therapeutic applications. Physiol. Rev. 86: 583-650.

Tertil M, Skrzypek K, Florczyk U, Weglarczyk K, et al. (2014). Regulation and novel action of thymidine phosphorylase in nonsmall cell lung cancer: crosstalk with Nrf2 and HO-1. PLoS One 9: e97070. 
Vardiman JW, Thiele J, Arber DA, Brunning RD, et al. (2009). The 2008 revision of the World Health Organization (WHO) classification of myeloid neoplasms and acute leukemia: rationale and important changes. Blood 114: 937-951.

Wang YC, Du X, Geng SX, Li YY, et al. (2011). Detection of methylation levels of multi-genes by real-time PCR in patients with myelodysplastic syndrome. Zhonghua Xue Ye Xue Za Zhi 32: 254-258.

Zhang Y, Song LX, Wu LY, Chang CK, et al. (2013). p15 (INK4B) methylation on prognosis and response to decitabine in patients with MDS. Zhonghua Xue Ye Xue Za Zhi 34: 237-241. 